\title{
ARQUITECTURA EN EL PAISAJE. NATURALEZA EN LA ARQUITECTURA
}

\author{
ARCHITECTURE IN THE LANDSCAPE. \\ NATURE IN THE ARCHITECTURE
}

\author{
María del Mar LozAno BARTOLOZZI \\ Universidad de Extremadura. España \\ ORCID: 0000-0001-5457-7890 \\ marlbart@unex.es
}

En el artículo se analizan algunas construcciones de arquitectura contemporánea realizadas en poblaciones de la provincia de Badajoz, que ofrecen la particularidad de integrar la naturaleza o entrar en diálogo con el paisaje natural circundante, además de ser muestra de proyectos originales e innovadores en distintos aspectos.

Palabras clave: arquitectura; naturaleza; paisaje; Badajoz; siglo XXI.

The paper analyzes some contemporary architecture constructions made in towns of the province of Badajoz, which offer the particularity of integrating nature or entering into dialogue with the surrounding natural landscape, in addition to being a sample of original and innovative projects in different aspects.

Keywords: architecture; nature; landscape; Badajoz; $21^{\text {st }}$ century.

\section{INTRODUCCIÓN}

En las primeras décadas del siglo XXI algunas poblaciones y enclaves rurales de la provincia de Badajoz se han visto transformados en su paisaje urbano y periurbano por determinadas edificaciones, introducidas tanto en sus centros históricos como en espacios de la periferia. La integración de la naturaleza en las mismas es una preocupación generalizada. Además, a veces intentan un diálogo 
con el entorno urbano y otras con el inmediato paisaje, o simultáneamente con ambos. Se trata de obras en variados contextos ${ }^{1}$.

Hablamos de proyectos innovadores y específicos entre los que destacan en particular los realizados por arquitectos de talante renovador que han sido galardonados con premios de reconocimiento regional, nacional e internacional. Son proyectos que se han propuesto para colaborar en los objetivos de desarrollo local. Queremos por ello analizar algunas piezas construidas y la relación con el espacio donde se ubican, para insistir en la interacción que han buscado y logrado en la configuración del paisaje del entorno, así como en sus cualidades formales y otros aspectos de planificación y sociología urbana para contribuir a la ordenación del espacio. Muchos de ellos se sitúan en los bordes y las periferias de las poblaciones y son los que más nos interesa comentar en este trabajo. También hay que señalar que en algunos observamos cómo no solamente tienen en cuenta el diseño sino que tratan de resemantizar las formas y el sentido del proyecto en la relación con el medio ambiente.

Nos vamos a referir al Museo de las Ciencias del Vino de Almendralejo, que supuso una operación integradora en una fábrica ya arruinada de alcoholes, con la rehabilitación, así como la ampliación, del inmueble para los nuevos usos museísticos. Enlaza urbanísticamente con la plaza de toros situada enfrente y el parque de la Piedad. Resuelve un conjunto de ocio, con la recuperación de la memoria y la identidad de la actividad vitivinícola, sin renunciar a las posibilidades de una arquitectura moderna y neo-racionalista. Se añade que supuso un hito más en la consolidación de una de las chimeneas de ladrillo de las que disfruta la población. Ya anteriormente se afianzó la chimenea de la fábrica de harina -antiguo convento de San Antonio-, rehabilitada para centro cultural en el mismo Almendralejo. Hablamos, por tanto, de rehabilitación y de nueva arquitectura.

Otro inmueble a comentar es el Palacio de Congresos y Exposiciones Vegas Altas, de Villanueva de la Serena, una incorporación exnovo de un hito arquitectónico en un borde de la población que sobresale en el panorama urbano/rural, con irrupción atrevida y desafiante: "Un edificio exento, flotante en el campo, como una bala gigantesca de heno"2. Lo mismo ocurre con el Palacio de Congresos de Plasencia, construido por José Selgas y Lucía Cano en un extremo de la

${ }^{1}$ Este trabajo se enmarca dentro del Proyecto de Investigación Nacional del Ministerio de Ciencia, Innovación y Universidades del Gobierno de España titulado "Paisajes Culturales en la Extremadura Meridional: una visión desde el Patrimonio" (HAR201787225-P), cofinanciado por los fondos FEDER.

2 Archivo del Servicio de Obras y Proyectos, Consejería de Cultura, Turismo y Deportes, Junta de Extremadura (ASOP), Pancorbo Crespo/Villar Martínez: Palacio de exposiciones y congresos "Vegas Altas". Villanueva de la Serena. Memoria descriptiva, Proyecto básico y de ejecución y estudios complementarios. https://www.jesusgranada.com/palaciode-congresos-y-exposiciones-vegas-altas (26-09-2019). 
población, insertado en un paisaje con berrocales graníticos, al que no nos vamos a referir ahora. Son obras que responden a la política de equipamientos de ocio, gestión cultural y de negocios, turística y profesional con nuevas tecnologías y espacios para el encuentro, como estrategia de desarrollo local y comarcal, en una región donde en pocos años han surgido los Palacios de Congresos y Exposiciones de Mérida (2004), Badajoz (2006), Cáceres (2011) y los mencionados de Plasencia (2017) y Villanueva (2017).

Otro edificio que aporta una arquitectura simbólica pero diferente a la tradición del hábitat del entorno es el Centro Integral Territorial de la Mancomunidad de Olivenza en dicha población frontera con Portugal. Arquitectura y naturaleza se integran en el mismo y los espacios llenos y vacíos se relacionan con ánimo de lograr transparencias, así como la circulación del exterior en la mirada del interior.

También nos interesa mencionar una construcción dedicada al deporte y encajada en el borde de un embalse sin perturbar el entorno, con vistas a la propia naturaleza: el Pabellón de Remo de Alange (Badajoz), situado frente al pantano del mismo nombre. Está dotado, además, de la capacidad de ser un mirador, cercano al llamado mirador Pata del Buey, un pétreo abrigo natural en un espacio con caprichosas rocas, que constituye un lugar privilegiado y ajardinado.

\section{ALMENDRALEJO, MUSEO DE LAS CIENCIAS DEL VINO (MUCVA) (2006-2009)}

La localidad de Almendralejo en la Baja Extremadura mantiene la tradición de su industria alcoholera y producción de vinos. En función de la misma, cuando se organizaron una serie de Museos de Identidad - desde el gobierno autonómico socialista-, que pretendieron determinados objetivos, entre los cuales estaba mantener la memoria de edificios y de algunas actividades locales ${ }^{3}$, uno de ellos fue el Museo del Vino de esta población. La obra salió a concurso y fue ganada por el estudio de los arquitectos pacenses Daniel Jiménez y Jaime Olivera ${ }^{4}$, que hicieron el proyecto y la dirección de obra, en la cual también intervino la arquitecta Beatriz Martínez . El promotor fue la Consejería de Cultura y Turismo de la Junta de Extremadura. La actuación consistió en la rehabilitación de una antigua alcoholera comprada por el Ayuntamiento de Almendralejo, con el añadido

${ }^{3}$ Asensio/Caldera/Pol Méndez, 2010: 49-81.

${ }^{4}$ Los arquitectos Daniel Jiménez y Jaime Olivera han realizado otras obras de indudable interés al ganar varios concursos, como el Servicio de emergencias 112 de Extremadura en Mérida, las Bodegas Habla de Trujillo, la Casa JJP de la Urbanización Las Vaguadas (Badajoz), el Centro I+D+i de la sostenibilidad local de Badajoz, el edificio ITV Olivenza, el albergue rural en la Vía de la Plata en Casar de Cáceres, etc. Jiménez/Olivera, 2018: s. p.

${ }^{5}$ Con un presupuesto de 1.300 .000 euros. 
de nuevas partes construidas (Figura 1). Una superficie total de $3.025 \mathrm{~m}^{2}$. El edificio se encuentra cercano a la carretera Nacional 630, junto al parque de la Piedad, donde se ubica el Santuario de Nuestra Señora de la Piedad, patrona de la población, y frente a la plaza de toros, que ofrece la peculiaridad de albergar bajo las gradas una bodega con una serie de conos para el vino ${ }^{6}$. También está cercano el Museo Devocional.

La obra que comentamos es una intervención de especial interés por su diálogo con el patrimonio. Un patrimonio etnográfico e industrial, a través de su valorización ${ }^{7}$. Otras actuaciones en esta línea en la región han sido la rehabilitación de una antigua fábrica de harinas para Casa de Cultura en Villafranca de los Barros, o el edificio del Embarcadero en las minas de Aldea Moret en Cáceres, rehabilitado para vivero de empresas y asociaciones ciudadanas, ocio y tiempo libre, y servicios generales.

El programa arquitectónico de Almendralejo utiliza el contenedor de una antigua alcoholera de mediados del siglo XX. El exterior es muy sobrio, de color blanco, propio de la Baja Extremadura, con escasos vanos y trazado rectilíneo un tanto neo-racionalista para organizar un recorrido expositivo en cinco salas $^{8}$ y dos plantas, del que forma parte el propio edificio. Se han aprovechado los depósitos originales de vino y alcohol ${ }^{9}$. Además, en el mismo, hay una serie de espacios para los servicios de administración y almacenes. Se añaden elementos adosados que envuelven en parte el antiguo inmueble. Así mismo tenía un patio interior para maniobras que ahora se ha convertido en un elemento de la exposición como jardín oculto con un huerto de viñas -es el denominado jardín de viñas, donde se plantaron distintos tipos de las mismas con diferentes variedades de uvas de la zona-, que sirve a fines didácticos para poder observar distintas especies, prácticas de la poda, etc., más la posibilidad de hacer allí actividades al aire libre y servir de terraza y auditorio. La cubierta es de zinc con un moderno tratamiento. Como afirmaron los arquitectos Tuñón y Mansilla al comentar este proyecto arquitectónico y museístico: "Es precisamente esa geometría de «comportamiento» orgánico, es decir, orgánico en su forma de proceder, la que logra imaginar unos espacios «entre los espacios», un conjunto de lugares que se traban con los jardines, y con la naturaleza, donde el concepto de transparencia se expande, y nos interroga sobre el sentido tradicional de las palabras

${ }^{6}$ Lozano Bartolozzi/Bazán de Huerta, 1990: 179-205.

7 Granada, 2010. https://www.jesusgranada.com/museo-vino-almendralejo-jimenezferrera-martinez-olivera (25-09-2019).

${ }^{8}$ Dedicadas a la Arqueología, Campo, Bodega, Ciencias del Vino y Alcoholera.

${ }^{9}$ http://www.museovinoalmendralejo.com/el_museo.php (25-09-2019). 
exterior e interior... unos espacios que esperamos pronto poder disfrutar, en vivo y en directo" 10 .

Los autores del proyecto han escrito que las nuevas dotaciones se plantearon como un crecimiento natural orgánico en torno a la crujía original del edificio por lo que huyeron de un estricto lenguaje racional o funcionalista: "la ampliación adopta una geometría en crecimiento, sin simetrías ni orden previo, atenta a situarse según el sol o la humedad, o la distancia a los demás apéndices, tal como lo haría la rama de un árbol. Mediante esa expansión orgánica, el edificio coloniza el patio entrelazando llenos y vacíos. Transforma el modo de producir la arquitectura ya que inserta espacios intersticiales, un conjunto de lugares trabados con el jardín" 11 .

En el conjunto destaca la conservación de una tradicional chimenea de ladrillo, pues, como ya se ha señalado, el paisaje urbano de Almendralejo está marcado por algunas chimeneas de sus antiguas industrias: "Almendralejo está salpicada de chimeneas de ladrillo, algunas de las cuales fueron construidas por maestros valencianos. Esta influencia levantina se refleja en las características de otras chimeneas de la zona, cuya tipología estándar era la de chimenea de sección octogonal y circular. Pero abunda la diversidad de estilos y podemos encontrar en la ciudad chimeneas de distinta altura, construidas para diferentes tipos de industria" 12

Felizmente existe en la población una asociación denominada "Amigos de la chimenea. Patrimonio Cultural de Almendralejo", y una voluntad de protección urbana de las mismas al incluirlas en el futuro catálogo de bienes protegidos del futuro Plan General Municipal (PGM) ${ }^{13}$.

Exteriormente el edificio es bastante compacto. Ofrece un enfoscado blanco que recuerda las paredes exteriores que tenía el edificio original enjalbegado. Los elementos nuevos, como auditorio, corredor y distribuidor del jardín, etc., se construyen en acero realizado artesanalmente, por lo que es claramente diferenciador de la obra original. Destaca el uso del espacio y de la luz.

La musealización incide en distintos recursos: los habituales de cartelería, objetos propios de carácter etnográfico y elementos de tipo antropológico (Figura 2). Se añade un juego de sensaciones interactivas a través de la percepción de aromas, sabores, sonidos, así como del color, tan importantes en las catas enológicas de los vinos. El objetivo es mostrar la historia identitaria, así como poner en evidencia la innovación relacionada con el cultivo del vino. Hablamos de un

${ }_{10}$ Texto de una conferencia de los arquitectos Tuñón y Mansilla, del año 2006, facilitado por el estudio Jiménez y Olivera.

11 AA.VV., 2019: 104.

12 Díaz, 2017: 39.

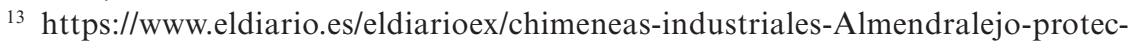
cion-legal_0_945606406.html (26-09-2019). 
proyecto realizado para contribuir al desarrollo local, pero también de un museo integrado en la propia promoción de la actividad vitivinícola de la comarca circundante de la Tierra de Barros, teniendo en cuenta la importancia y el desarrollo de la misma en las últimas décadas. No falta una tienda y diversas actividades relacionadas con concursos de cata, conciertos, etc. Pertenece a la Asociación de Museos del Vino de España. La rehabilitación fue premiada en los Prix européen d'architecture Philippe Rotthier de Bruselas.

\section{CENTRO INTEGRAL TERRITORIAL DE LA MANCOMUNIDAD DE OLIVENZA (CONSTRUCCIÓN EN 2010)}

En el suroeste de Extremadura se encuentra la localidad fronteriza de Olivenza, cercana a la vega del río Guadiana y el puente de Ajuda. Es un gran conjunto histórico artístico y medioambiental, donde es importante su recinto amurallado, sus iglesias y edificios del Antiguo Régimen, pero también la arquitectura de la primera mitad del siglo XX con firmas de buenos arquitectos municipales y provinciales como Francisco Vaca o Donato Hernández y los distintos ensanches y el planeamiento urbano producidos en el citado siglo ${ }^{14}$. En uno de los extremos periféricos de la población, alejado del cinturón de las excepcionales murallas tipo Vaubán, donde se encuentra una zona de expansión urbana dotacional con viales de gran regularidad y parcelas de distintos usos ${ }^{15}$, como una urbanización de viviendas adosadas y un parque público, se encuentra este edificio encargado por la Diputación de Badajoz ${ }^{16}$ y proyectado como un nuevo hito por el mismo estudio citado de Daniel Jiménez y Jaime Olivera, junto a otros edificios sin una personalidad particular, que ha buscado referencias en la propia población y su patrimonio. La superficie es un área de $1.150 \mathrm{~m}^{2}$. Fue inaugurado en diciembre de 2013.

Los servicios que presta son los referentes a la administración de la Mancomunidad Integral de Olivenza -formada por 10 municipios-, una de las unidades administrativas territoriales de tipo comarcal de la provincia de Badajoz. El edificio, de un gran volumen concentrado, llama especialmente la atención. Es una pieza particular formada por elementos geométricos rectangulares y cerrados que

14 Pizarro Gómez, 2005: 99.

15 En el año 2005 fue aprobado el Plan General Municipal de Olivenza.

$16 \mathrm{https} / / / \mathrm{www}$.europapress.es/extremadura/noticia-nuevo-centro-integral-territorialcomarca-olivenza-servira-punto-encuentro-empresarios-zona-20131216152906.html (2809-2019): “El nuevo Centro Integral Territorial 'Comarca de Olivenza' servirá de punto de encuentro para empresarios de la zona... Este proyecto está promovido por el Área de Fomento, Obras y Asistencia Técnica a Municipios; el Área de Planificación y Nuevas Tecnologías y el Área de Igualdad y Desarrollo Local de la Diputación y financiado en un 70 por ciento por el Fondo Europeo de Desarrollo Regional (Feder)". 
parece flotar sobre una base de segmentos inclinados y reducida cimentación, con planos triangulares de distintos entrantes y salientes (Figura 3). La mirada, conforme nos acercamos, nos descubre provocativamente un inmueble de estilo más cercano a un revival "brutalista" arquitectónico de los años cincuenta que a la arquitectura tradicional del mundo rural, si bien la lectura del mismo realizada por Jesús Granada ${ }^{17}$ nos aproxima a distintas referencias propias del entorno e incluso a la arquitectura vernácula en detalles como el "fresco zaguán de la arquitectura extremeña". El material empleado en el exterior es oscuro y relacionado con la cercana sierra del Alor, por lo que se adquiere la apariencia de una roca o del elemento de una fortificación que evoca los cercanos baluartes y las torres del castillo interior. La estética recuerda a otros edificios contemporáneos internacionales, muy cerrados exteriormente y llenos de espacios abiertos y transparentes en el interior gracias a patios y jardines: "El CIT (Centro Integral Territorial) apuesta por referentes profundos, anacrónicos: la roca oscura de la montaña, los volúmenes de las torres defensivas, las piedras reunidas, juntas, en un campo labrado, el fresco zaguán de la casa extremeña" 18 .

Los usos para centro social local, a disposición de actividades del barrio como el encuentro entre empresarios, se extienden a un aparcamiento, jardín, locales en la planta baja con aula y sala de reuniones. Además han sido empleadas nuevas tecnologías con incidencia en la revalorización del medio ambiente por las instalaciones bioclimáticas. El interior es diáfano, luminoso y funcional con cubiertas ajardinadas muy ecológicas (Figura 4): "El espacio nunca es neutro, pero en el proyecto que proponemos la sencillez, quitar lo que sobra, quedarse en los huesos, es estrategia, no forma. La condición corpórea y táctil de la propuesta tiene algo de recuperación de situaciones originarias que, sin duda, estuvieron presentes en las obras de aquellos que por primera vez se enfrentaron a la construcción: un abrigo, una sombra, un camino, una atalaya, un lugar en la naturaleza...".

Haber incluido un jardín en el edificio, en un aterrazamiento exterior, es una apuesta presente en la arquitectura contemporánea que pretende participar de una visión ecologista o medioambiental. También en el edificio para la ITV que hacen los mismos arquitectos en Olivenza, incluyen un jardín en el espacio o patio abierto entre los elementos construidos que constituyen el inmueble en su totalidad. No olvidemos, sin embargo, la importancia de la naturaleza unida al funcionalismo que ya defendiera Alvar Aalto o el organicismo de Wright con la

${ }^{17} \mathrm{http}$ //hicarquitectura.com/2013/02/gap-arquitectos-centro-integral-territorial-deolivenza-badajoz/ (08-08-2019). Fuente: CAP Arquitectos. Fotografía: Jesús Granada.

$18 \mathrm{http}$ //hicarquitectura.com/2013/02/gap-arquitectos-centro-integral-territorial-deolivenza-badajoz/ (08-08-2019): "Sí hay control energético y abundancia de aislamiento. De inercia térmica. Hay instalaciones bioclimáticas, haciendo casi autosuficiente el edificio. Muros trombe, suelo refrescante y radiante, ventilación continua, cubiertas jardín ecológicas, aljibes...". 
búsqueda de la armonía entre la arquitectura y la naturaleza. O las terrazas ajardinadas en la arquitectura de Le Corbusier.

Otros ejemplos de distintos arquitectos preocupados por la integración de la vegetación son el Centro Cultural La Gota y Museo del Tabaco en Navalmoral de la Mata, edificio construido como espacio cultural que acoge la exposición permanente de la artista Feliú y el museo del tabaco. Ofrece una particular manera de acercarse a la naturaleza, tanto como evocación en la propia ordenación del edificio como al construir un jardín vertical con plantas de tabaco y otras especies propias de la zona. El título La Gota hace mención al edificio existente con anterioridad dedicado a una institución que ofrecía ayuda para la nutrición infantil. Sus autores son los arquitectos Losada y García, que mantienen estudio tanto en Navalmoral de la Mata como en San Diego (EE.UU.). Un nuevo ejemplo lo encontramos en el Conservatorio de Música de Almendralejo ${ }^{19}$. O la cubierta ajardinada del edificio Administrativo y Nuevas Titulaciones de la Universidad de Extremadura en Mérida, obra de Iñaki Ávalos, Juan Herreros, Javier Manso y María Ángel López Amado (1999-2001), situado fuera de la ciudad histórica y en la zona colindante con el río Guadiana, cuya vegetación de ginkgos biloba, que también se extienden por algunas zonas del edificio, tiene la finalidad de ser una forma de asilamiento de la radiación solar, reforzada también ${ }^{20}$.

En el Instituto de Enseñanza Secundaria de Villanueva de la Serena (20042005), de los arquitectos Gerardo Ayala Hernández, Marcos Ayala Cabo y Mateo Ayala $\mathrm{Cabo}^{21}$, cabe destacar la integración de vegetación con olivos en los diversos patios acristalados del edificio para proporcionar buenas vistas ${ }^{22}$. Se trata de un edificio cuyo proyecto ganó el concurso convocado por la Consejería de Educación de la Junta de Extremadura en 2004, si bien la obra se llevó a cabo (2005-2006) por funcionarios de la entidad promotora que no realizaron completamente el proyecto original ${ }^{23}$. Como los propios autores expresan, tuvieron en cuenta el lugar del emplazamiento en pleno campo ${ }^{24}$, el olivar allí existente y que la edificación añadiera a la propia educación impartida ese ambiente saludable y ecológico, tal como perseguía la educación de la Institución Libre de Enseñanza por ejemplo: "El solar se encontraba en medio de un olivar y frente a la sierra, y el proyecto conservaba parte de los olivos en el interior de nuestra parcela. La fachada principal del edificio se abría a la vista de la sierra lejana, con

19 Prieto Fernández/Díaz Recasens, 2005: 92-105.

${ }^{20}$ AA. VV., 2007: 48.

${ }^{21}$ AA. VV., 2006: 156.

${ }^{22}$ AA. VV., 2006: 58.

${ }^{23} \mathrm{http}$ ///www.arquitectosayala.com/portfolio/instituto-de-ensenanza-secundaria/ (02-10-2019).

${ }^{24}$ AA.VV., 2007: 58. 
orientación Norte, ya que se trataba de un pueblo de Extremadura muy castigado por el sol" 25 .

El problema es la falta de interés por mantener estos elementos que son, además, un toque decorativo, pero en el que creen poco los que gestionan los mismos a la luz de los resultados que hemos podido observar.

\section{PABELLÓN DE REMO DE ALANGE (2007-2009)}

El centro de tecnificación de piragüismo y remo en el pantano de Alange fue financiado por el Gobierno (Consejo Superior de Deportes) y la Junta de Extremadura, y convocado en concurso (proyecto básico y ejecución) por la Consejería de los Jóvenes y del Deporte en el año 2006. El concurso fue ganado por José María Sánchez García ${ }^{26}$, un arquitecto autor de diversas obras, algunas en la propia región ${ }^{27}$, premiado por la revista $A D$ Architectural Digest como arquitecto del año 2016. Se trata de una construcción de gran sencillez formal, como es habitual en su estudio de arquitectura. La finalidad era la creación de un espacio polivalente en relación con los deportes del pantano, donde desarrollar diferentes programas como formación, entrenamientos, vestuarios, dormitorios, embarcadero, campo de regatas, zona médico-deportiva o actos públicos con área de aparcamiento. La superficie es de $2.000 \mathrm{~m}^{2} \mathrm{La}$ construcción fue realizada por la empresa Procondal en los años 2008-2009.

En la memoria del proyecto, ya se afirma que una de sus principales estrategias del mismo es el respeto al paisaje, que está dotado de un gran valor medioambiental. Se ubica al sur de la población, en un terreno con un pronunciado declive, que fue resuelto con un aterrazamiento en el que han sido construidas viviendas adosadas unifamiliares, mientras que en una cota inferior, y con un desnivel mucho más pronunciado, se desarrolla la construcción de este pabellón, sin interferir las vistas desde la parte urbana (Figura 5).

Así mismo, al ofrecer el terreno ciertas dificultades por su irregularidad, el proyecto tuvo que adecuarse a estas circunstancias y, como es habitual en la obra de José María Sánchez, la construcción parte de unas formas de geometría acusada y de gran claridad. Sin embargo, se trata de una geometría irregular y complicada, condicionada ante los límites del solar a los que se adapta la planta por exigencias de la normativa urbana.

${ }^{25} \mathrm{http}: / / \mathrm{www}$. arquitectosayala.com/portfolio/instituto-de-ensenanza-secundaria/ (02-10-2019).

${ }^{26}$ Sánchez García, 2011: 511-516.

27 Autor, entre otras obras, del Centro de Innovación Deportiva de Guijo de Granadilla (Cáceres), de la intervención para adecuación del entorno del templo de Diana de Mérida o la Industria de Montajes Eléctricos en Don Benito. https://www.jmsg.es/ 
La edificación consiste en un gran basamento o plataforma de hormigón, que a su vez está dotado de un gran mirador en sombra que permite contemplar abiertamente el paisaje, sobre la que apoya una cubierta muy ligera, totalmente transparente y abarcable visualmente, con una estructura de cerchas metálicas que no lo cubre en su totalidad, encintada en un plano interior por cristales. $\mathrm{Cu}$ bierta definida por el arquitecto como una "pieza industrial" aunque "delicada", que supone un enlace "con la tradición constructiva propia de las explotaciones agrícolas y la pequeña industria local".

Los materiales utilizados para la misma son elementos prefabricados y perfiles estandarizados de acero, o piezas que se ensamblan y despiezan de forma sencilla, "sin revestimiento y enfatizando la técnica empleada". En el borde de la plataforma hay una "grada perimetral excavada que evita la colocación de una barandilla convencional y permite el seguimiento de las competiciones de remo, consiguiendo una continuidad visual con el paisaje". En el solado de esta estructura metálica, se abre hacia el interior de la construcción de hormigón un amplio hueco que conecta con la sala principal del proyecto iluminada por la amplitud de aquél y, en el interior del mismo, "un espacio diáfano y de uso flexible de 21 x 21 $\mathrm{m}$ a modo de plaza donde se desarrolla el programa principal. Su dimensión viene dada por el radio de giro necesario para operar con las canoas" 28 .

Dentro del edificio, además de cumplir con las necesidades fundamentales, hay un programa secundario "en el espacio intersticial entre la sala central y el límite del edificio. En estos recintos perimetrales, resultado de la geometría irregular dada por el solar; se localizan las habitaciones, el gimnasio, los vestuarios y las oficinas".

Hay un doble acceso, uno en la planta baja para las embarcaciones y otro desde la plataforma superior a través de una espectacular rampa a la cota de 295 $\mathrm{m}$, que se desarrolla colgada mediante cables de acero inoxidable y pasamanos de acero galvanizado (Figura 6).

La obra ha sido valorada por su funcionalidad y, al mismo tiempo, por su relación con el paisaje: "Ricorda una pietra miliare capace di misurare metafóricamente la distanza che intercorre tra l'architettura e il paesaggio che la accoglie" 29 .

Sin embargo, la gestión ha sufrido una serie de avatares y problemas, pues una vez terminada la obra ha tardado mucho en ser utilizada. La Federación Extremeña de Piragüismo, a quien se le propuso la cesión de uso en el año 2012, no admitió la misma por los problemas existentes, como el hecho de no estar urbanizada la zona y faltar algunos elementos como el embarcadero. Posteriormente, se harán nuevas inversiones y mejoras en la urbanización del entorno y equipamiento del conjunto (2016). Finalmente, en el año 2018, se firma un convenio entre Junta y Ayuntamiento de Alange con la cesión al mismo por diez años, de tal

\footnotetext{
${ }^{28}$ Sánchez García, 2017: 18-27.

${ }^{29}$ Ferrari, 2014: 25.
} 
forma que el Consistorio saque a concurso su gestión. En estos momentos está pendiente de su inauguración con el uso para gimnasio en la parte superior y las actividades deportivas previstas en el interior ${ }^{30}$.

Este edificio es sencillo y realizado en hormigón ${ }^{31}$. Una obra distinta formalmente pero con planteamientos conceptuales parecidos, a la que el mismo arquitecto realizó en una pequeña península del embalse de Gabriel y Galán en el término de Guijo de Granadilla (Cáceres): el Centro Internacional de Innovación Deportiva en el medio natural El Anillo. Una arquitectura que analizamos Javier Cano y yo misma en un artículo denominado "Arquitecturas que miran al agua" ${ }^{32}$. En una y otra conviven la geometría construida y el territorio natural ${ }^{33}$.

\section{PALACIO DE CONGRESOS VEGAS ALTAS DE VILLANUEVA DE LA SERENA (2010-2014, 2017).}

El proyecto aborda una actuación de nueva planta en la que el entorno periférico al este de esta población de 26.000 habitantes es urbanizado según un Plan Parcial del PGOU ${ }^{34}$, adaptándose a una topografía desigual. La ordenación se hace con una gran plaza inclinada y un jardín de diseño con formas geométricas abstractas o parque público, añadiendo una construcción semienterrada. El conjunto crea un fragmento de paisaje cultural en un espacio que, si por una parte forma borde con un vial y el acceso al conjunto urbano de la ciudad, por otra parte se integra al mismo tiempo en el campo o paisaje natural de las Vegas Altas, en el paisaje de la Serena, creando una huella de nueva traza.

Desde un lateral de dicha plaza, se penetra bajo rasante al conjunto construido, que es iluminado por medio de lucernarios; mientras que un volumen cúbico que se convierte, al igual que el mencionado jardín, en una superficie de arte visual por los juegos geométricos de sus diversos huecos de perfil desigual y las posibilidades cromáticas, sobresale en altura (Figura 7). En su interior se encuentran la caja escénica y la concha acústica del auditorio principal que tiene un aforo de 800 personas. La oficina Pancorbo Arquitectos S.L.P. ganó el primer premio del concurso público para la redacción del proyecto promovido por la Consejería de Cultura y Turismo de la Junta de Extremadura en el año 2008 como elemento de recurso turístico, de ocio y de negocios con posibilidad de incidencia en la Mancomunidad o Comarca de las Vegas Altas del Guadiana, La

\footnotetext{
30 López Lago, 2019.

31 Sánchez García, José María: Memoria del Proyecto.

32 Lozano Bartolozzi/Cano Ramos, 2012: 137-144. Biagi, 2010: 69-76.

33 En ambos casos, "la superposición de trazas - preexistentes y propositivas-genera una nueva realidad más compleja”. Sánchez García, 2017: 20.

${ }^{34}$ La parcela se encuentra incluida en el Plan Parcial del Sector 2 "Corona Este" del PGOU de Villanueva de la Serena.
} 
Serena y la Siberia. Los arquitectos que trabajaron en el mismo fueron Luis Pancorbo, José de Villar, Carlos Chacón e Inés Martín Robles ${ }^{35}$. La superficie total es de $5.962,75 \mathrm{~m}^{2}$. Bajo rasante $5.213,60 \mathrm{~m}^{2}$ y sobre rasante $749,15 \mathrm{~m}^{2}$. Los usos como edificio polivalente con auditorio y lugar de celebración de congresos dan lugar también a distintas dependencias con oficinas y restaurante ${ }^{36}$. Las propias condiciones del pliego técnico del concurso incidían en aspectos como: "Las propuestas presentadas se ajustarán a las actuales tendencias arquitectónicas, buscando soluciones atemporales de carácter exclusivo, que ante todo sean innovadoras, originales, y capaz de marcar un antes y un después en la historia arquitectónica de la ciudad" 37 .

A esto se añade la importancia para la buena acústica, aprovechamiento de luz y ventilación natural, ahorro energético, usos expositivos, urbanización y ajardinamiento exterior, etc. Por su clarividencia no podemos renunciar a reproducir algunas frases de la memoria que conceptualizan la propuesta teórica y constructiva del conjunto: "Se divide en dos volumetrías contrapuestas: un anillo plano semienterrado que se adapta al terreno y a los límites de la parcela, y otra cúbica que se eleva con rotundidad escondiendo el peine del teatro. La primera pierde su carácter de edificio y pasa a ser un campo un parque de la ciudad, con las franjas de lucernarios rasgando su superficie. La segunda refuerza su carácter de edificio exento al ser un cubo perfecto, perfecta figura de un hito". "Por un lado, un volumen es callado, pretende pasar desapercibido, el otro se ilumina y es escaparate de todos los eventos que en el transcurren, visible desde todas partes, un faro en el mar del campo de la Vega"38 (Figura 8).

Los materiales también contribuyen a su originalidad y diversas texturas, por ejemplo el cubo tiene una fachada interior "portante de hormigón de color dorado y perforada por grandes huecos de forma irregular y otra, ligera formada por un trenzado de maromas de color dorado (forradas con una doble piel plástica para asegurar su estabilidad ante agentes atmosféricos) que a modo de envoltorio recubre todo el cubo y protege de la excesiva insolación a los huecos

${ }^{35} \mathrm{https} / / / \mathrm{www}$. pancorboarquitectos.com/pec (03-10-2019).

${ }^{36} \mathrm{El}$ edificio dispone de un auditorio principal con capacidad para 800 espectadores y otro secundario con capacidad para 275 espectadores. Está dotado así de una Sala de Cámara para acoger espectáculos de música, teatro o conferencias. La sala polivalente se puede utilizar para recepciones, exposiciones temporales o como galería, y el cubo se completa con camerinos. Las características del conjunto pueden consultarse en https://villanuevadelaserena.es/palacio-congresos/ (03-10-2019).

37 ASOP, Pancorbo Crespo/Villar Martínez: Palacio de exposiciones y congresos "Vegas Altas". Villanueva de la Serena. Memoria descriptiva, Proyecto básico y de ejecución y estudios complementarios.

38 ASOP, Pancorbo Crespo/Villar Martínez: Palacio de exposiciones y congresos "Vegas Altas". Villanueva de la Serena. Memoria descriptiva, Proyecto básico y de ejecución y estudios complementarios. 
interiores dando un aspecto de bala de paja en el paisaje". Estas cuerdas ofrecen una epidermis ligada al entorno rural. Las cubiertas están ajardinadas.

El estudio Pancorbo tiene muy presente la vegetación en sus construcciones y los espacios que genera la arquitectura en su entorno, ejemplo es el Centro Cultural El Jesús en el Ayuntamiento de Santa Eulalia del Río en Ibiza (2010), donde también ha tenido en cuenta el espacio público al insertar este centro de ocio con la configuración de un paisaje ajardinado y de flujos peatonales alrededor.

\section{CONCLUSIONES}

Consideramos que a lo largo de este artículo se ha puesto en evidencia cómo la preocupación por integrar la naturaleza es común en las construcciones estudiadas. Así mismo, que dos actitudes diferentes se han producido en los proyectos analizados: la creación de hitos de referencia en el perfil o skyline del entorno o la mimetización con el mismo. Por lo tanto, la relación con el lugar es fundamental en todas las obras.

Se añade la utilización de nuevos materiales, el diseño y la expresión gráfica de las formas ofreciendo una intención de modernidad e innovación. El manejo de la luz, tanto natural como artificial, supone la creación de espacios metafóricos. También comprobamos la preocupación por la carga histórica con su memoria identitaria que puedan tener y por la carga medioambiental en los mismos, así como por la incidencia en el paisaje.

Son obras adjudicadas a través de concursos a los que han acudido los estudios de arquitectura y su visibilidad es una preocupación de los arquitectos que las muestran en sus páginas web con detallados y artísticos reportajes fotográficos que aumentan la lectura de las mismas como piezas que van del proyecto a la realidad con un discurso sugerente y sugestivo ${ }^{39}$. La valoración a través de los propios profesionales se ha manifestado en las Guías de Arquitecturas de Extremadura publicadas en los años 2007 y 2019. Sin embargo, el mantenimiento de esta arquitectura no se ha realizado conforme al proyecto inicial en todos los casos. Lamentablemente, en Olivenza, las cuidadas aportaciones de plantas y espacios verdes en las terrazas no se han atendido suficientemente y se vive de espaldas a ellos. Y el pabellón de remo de Alange espera todavía la utilización integral del mismo.

Fecha de recepción: 30 de octubre de 2019

Fecha de aceptación: 23 de febrero de 2020

${ }^{39}$ Puede consultarse https://www.jesusgranada.com/palacio-de-congresos-y-exposiciones-vegas-altas. Con fotografías de Jesús Granada y Roland Halbe. 


\section{BIBLIOGRAFÍA}

Asensio, Mikel/Caldera, Pilar/Pol Méndez, Elena (2010): "De los museos de identidad a los museos de mentalidad: bases teóricas de la recuperación de la memoria de los modernos museos de Extremadura". En: Museo: Revista de la Asociación Profesional de Museólogos de España, 15, pp. 49-81.

AA. VV. (2006): Arquitectura escolar. Madrid: Editorial Munilla-Leria. (2007): Guía de Arquitectura, 1975-2006. Badajoz: Junta de Extremadura/ COADE, tomo 1.

(2019): Guía de Arquitectura de Extremadura 2007-2017. Badajoz: Consejería de Sanidad y Políticas Sociales (Junta de Extremadura)/Colegio Oficial de Arquitectos de Extremadura, tomo 2.

Biagi, Marco (2010): "Centro tecnico sportivo a Guijo de Granadilla. José María Sánchez García”. En: Casabella, 785, pp. 69-76.

Díaz, José Luis (2017): "Skyline”. En: López Caballero, José Manuel/Fernández García, Atanasio (coords.): Cielos de Extremadura. Cáceres: Dirección General de Turismo/Fundación Xavier de Salas, pp. 36-39.

Ferrari, Massimo (2014): "José María Sánchez García-Astrazione e Paesaggio". En: Casabella, 838, pp. 25-29.

Granada, Jesús (2010): "Almendralejo Wine Museum: Daniel Jiménez + Jaime Olivera Arquitectos". En: Revista c3 Arquitectura, 309.

Jiménez, Daniel/Olivera, Jaime (2018): "Daniel Jiménez + Jaime Olivera-Arquitectos". En: Ayala Hernández, Gerardo (coord.): Encuentro/Encontros de Arquitectura. Badajoz: Real Academia de Extremadura de las Letras y las Artes, s. p.

López-Lago, J. (2019): "La Junta promete que este año abrirá el centro de piragüismo de Alange". En: Diario Hoy de Extremadura, 25-8-2019: <https:// www.hoy.es/extremadura/junta-promete-abrira-20190825000528-ntvo. html?ref=https:\%2F\%2Fwww.google.com $\% 2 \mathrm{~F}>$.

Lozano Bartolozzi, María del Mar/Bazán de Huerta, Moisés (1990): “Arquitectura pública en Almendralejo (1840-1940)”. En: Revista Norba-Arte, 10, pp. 179-205.

Lozano Bartolozzi, María del Mar/Cano Ramos, José Javier (2012): "Construcciones que miran al agua. Espejos patrimoniales en un paisaje intervenido por el Tajo". En: e-rph, 10, pp. 113-146.

Pancorbo, Luis/De Villar, José/Chacón, Carlos/Martín Robles, Inés (2015): "Palacio de Congresos. V. de la Serena (Badajoz)". En: AV Monografias, 173$174, \mathrm{pp}-54-63$.

Pizarro Gómez, Francisco Javier (2005): Paisajes Urbanos de Extremadura 2. Olivenza. Badajoz: Junta de Extremadura, Agencia Extremeña de la Vivienda, el Urbanismo y el Territorio. 
Prieto Fernández, Julián/Díaz Recasens, Gonzalo (2005): “Almendralejo. Conservatorio de Música”. En: Arquitectura Ibérica, 1 [11], pp. 92-105.

Sánchez García, José María (2011): "Centro de remo y piragüismo en Alange". En: Detail: revista de arquitectura y detalles constructivos, 5, pp. 511-516.

- (2015): "Centro de Remo en Alange (Badajoz)". En: AV Monografías, 173174, pp. 154-159.

(2017): “José María Sánchez García”. En: El Croquis, 189, pp. 4-97. (2018): “Dossier". En: AV Proyectos, 090, pp. 7-37. 


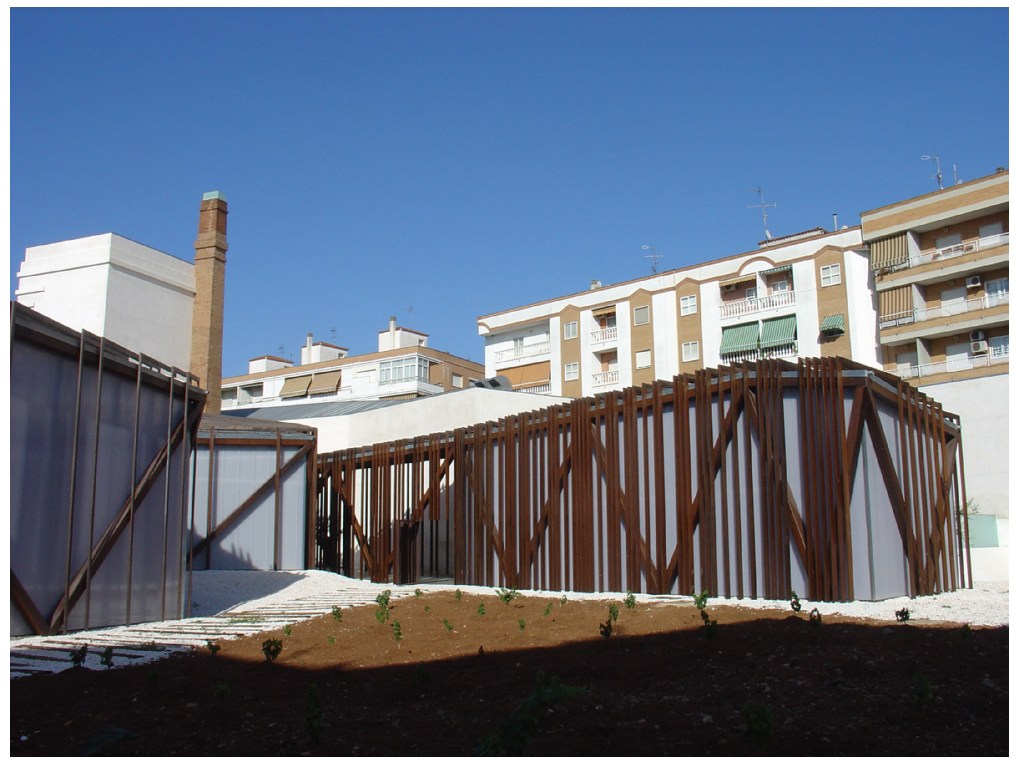

Figura 1. Daniel Jiménez, Jaime Olivera y Beatriz Martínez, Museo de las Ciencias del Vino, Almendralejo. Foto: María del Mar Lozano Bartolozzi.

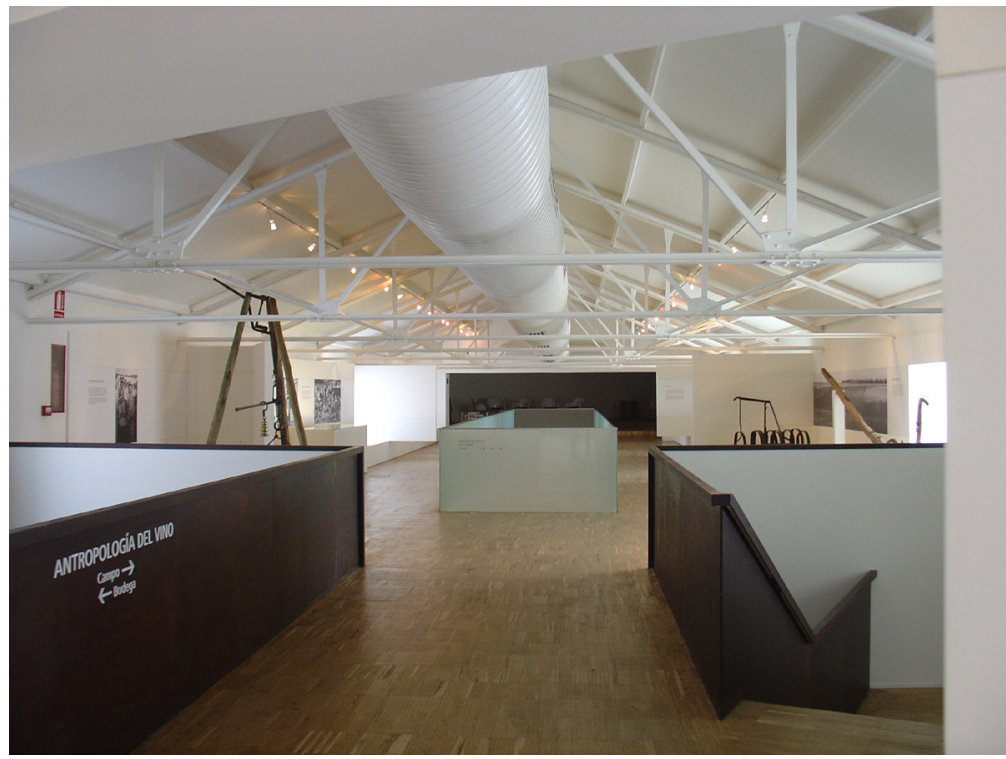

Figura 2. Daniel Jiménez, Jaime Olivera y Beatriz Martínez, Museo de las Ciencias del Vino, Almendralejo. Foto: María del Mar Lozano Bartolozzi. 


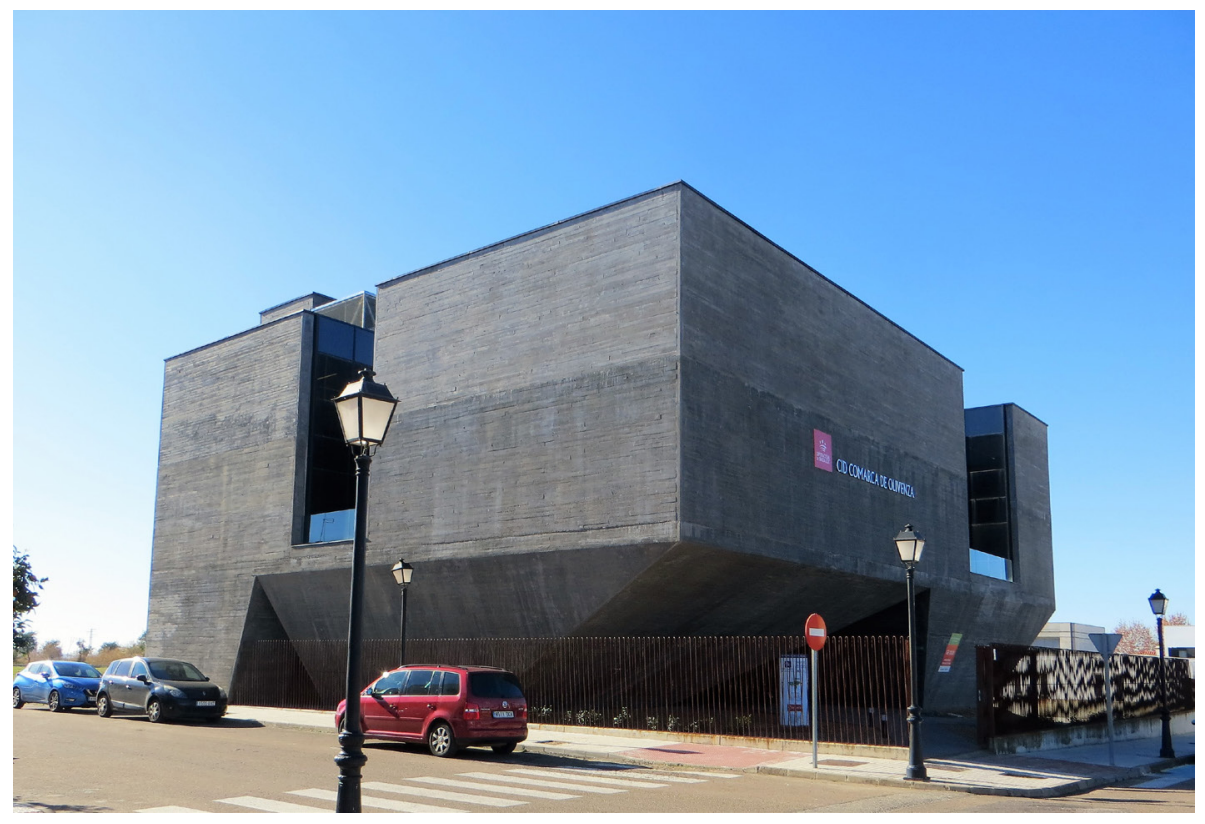

Figura 3. Daniel Jiménez y Jaime Olivera, Centro integral territorial de la Mancomunidad de Olivenza. Foto: María del Mar Lozano Bartolozzi. 


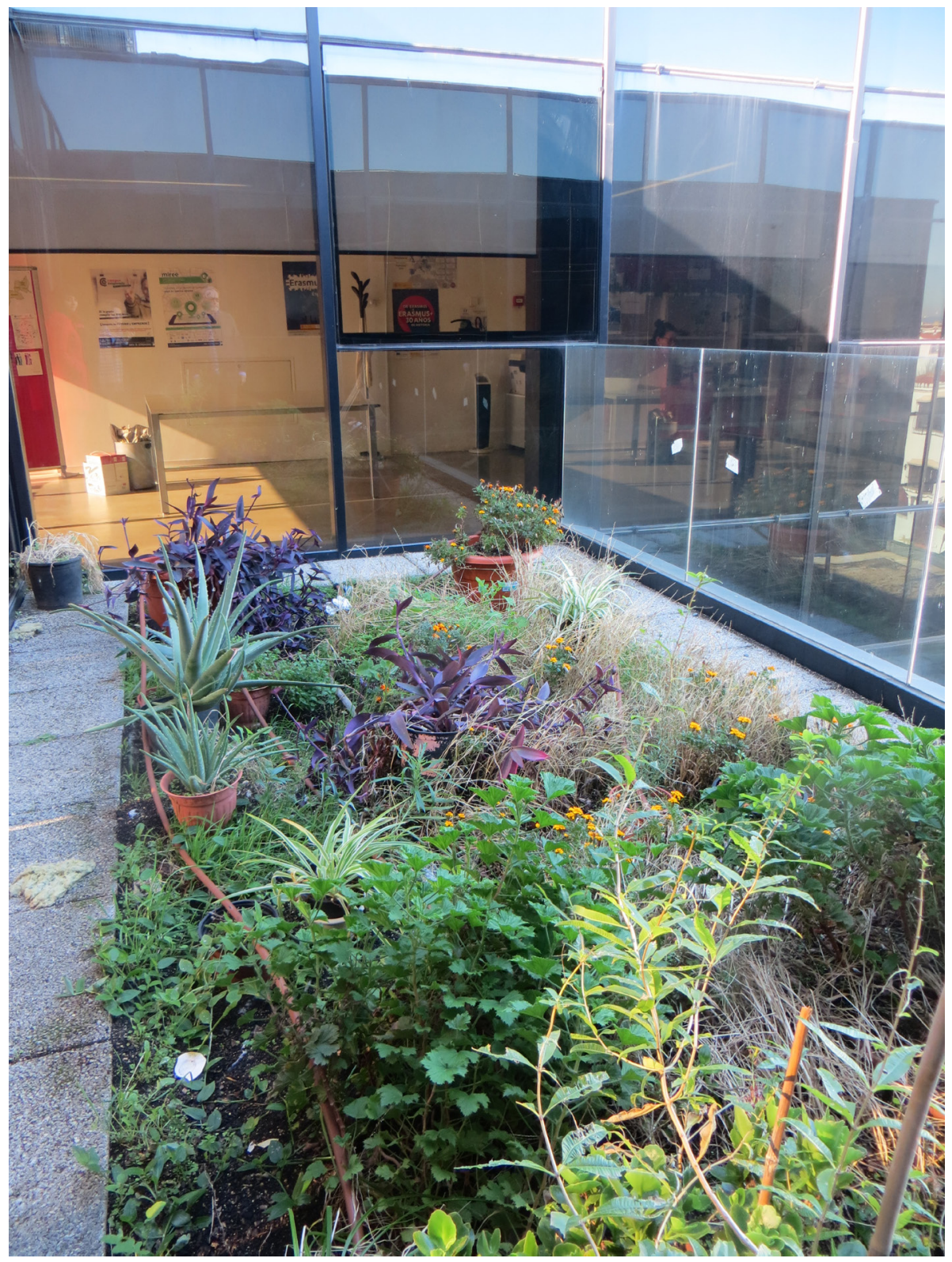

Figura 4. Daniel Jiménez y Jaime Olivera, Centro integral territorial de la Mancomunidad de Olivenza. Foto: María del Mar Lozano Bartolozzi. 

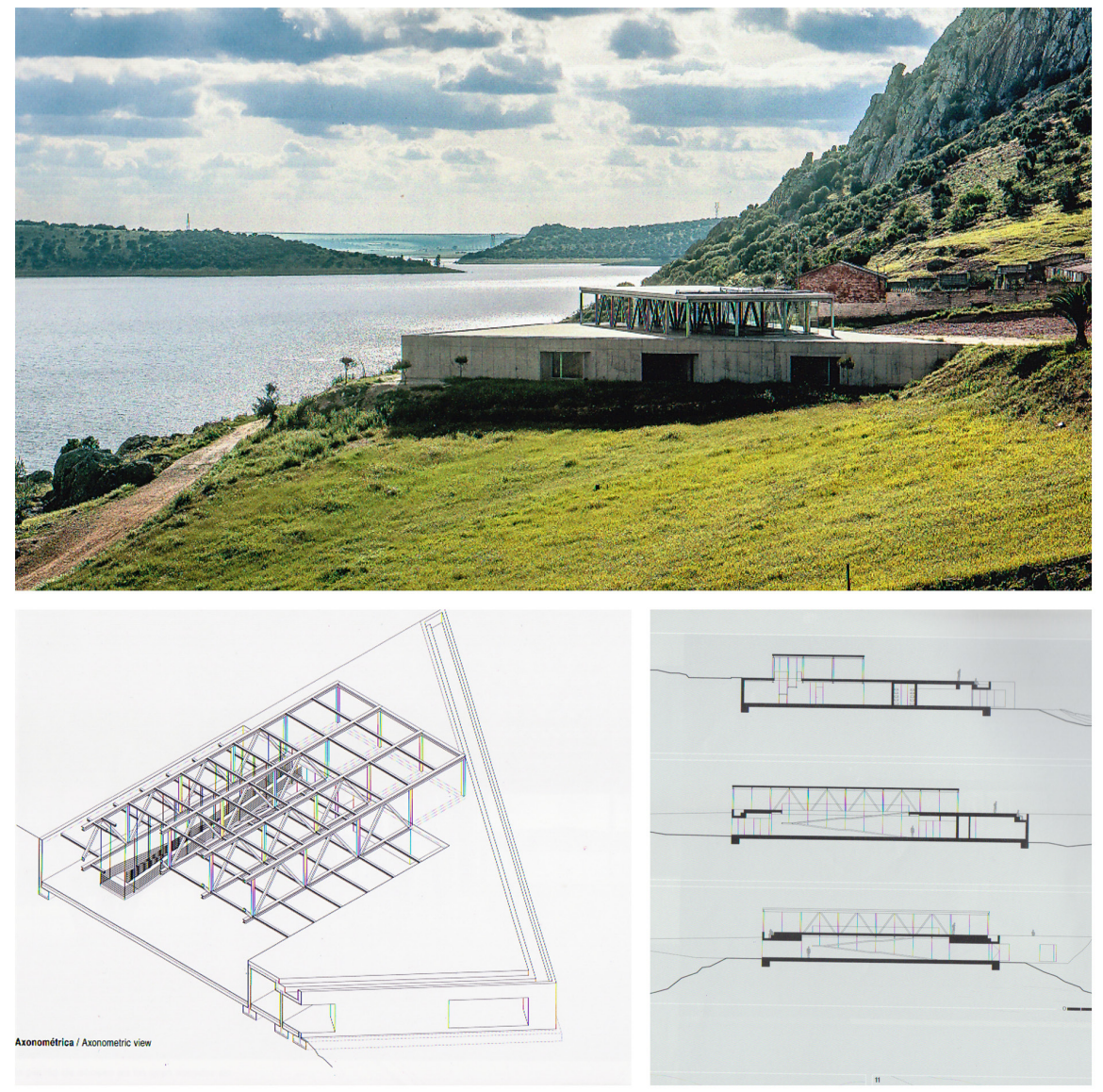

Figura 5. José María Sánchez García, Pabellón de remo de Alange (visión axonométrica). Foto y secciones: cortesía de José María Sánchez García. 

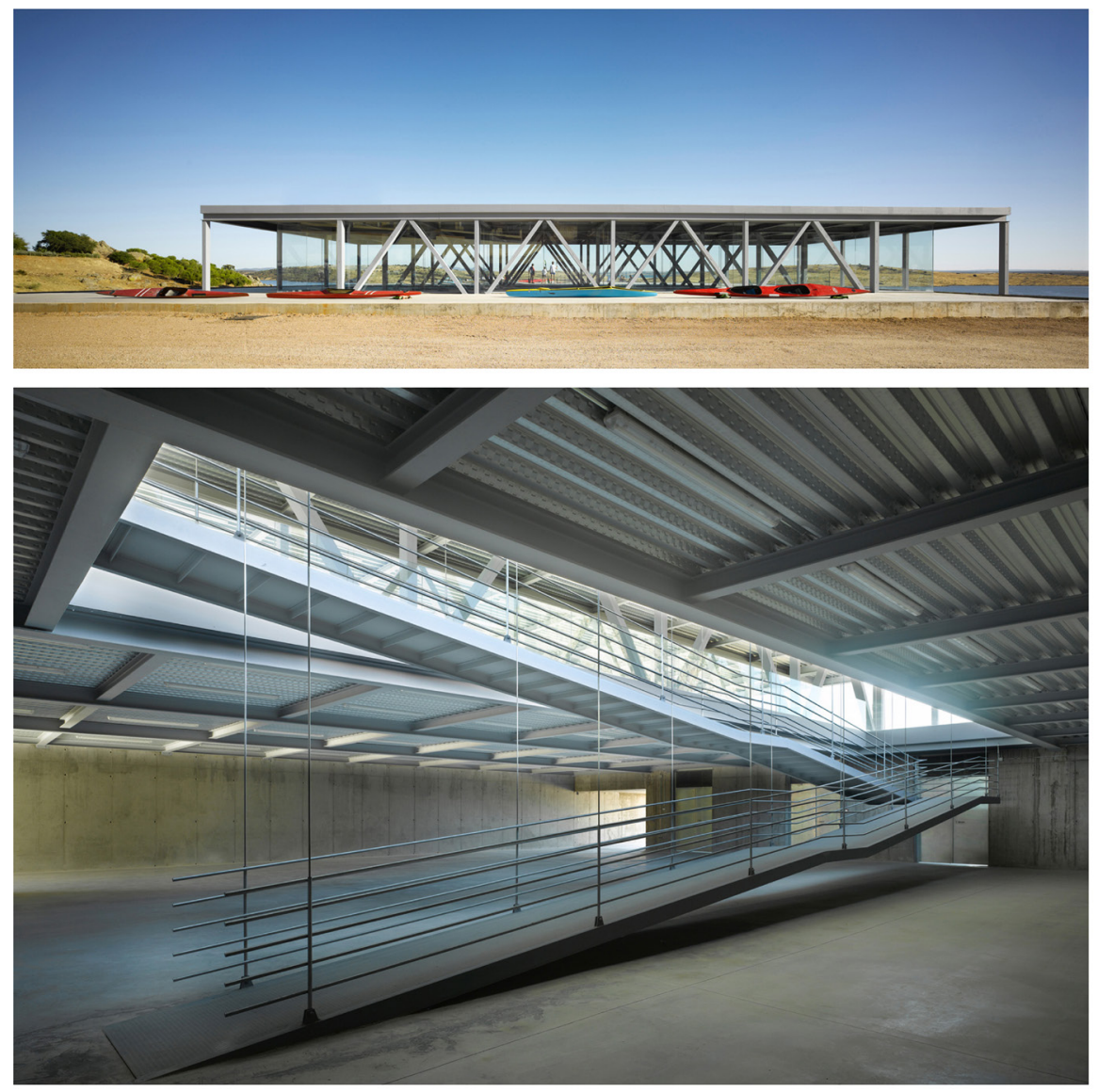

Figura 6.José María Sánchez García, Pabellón de remo de Alange. Fotos: cortesía de José María Sánchez García. 

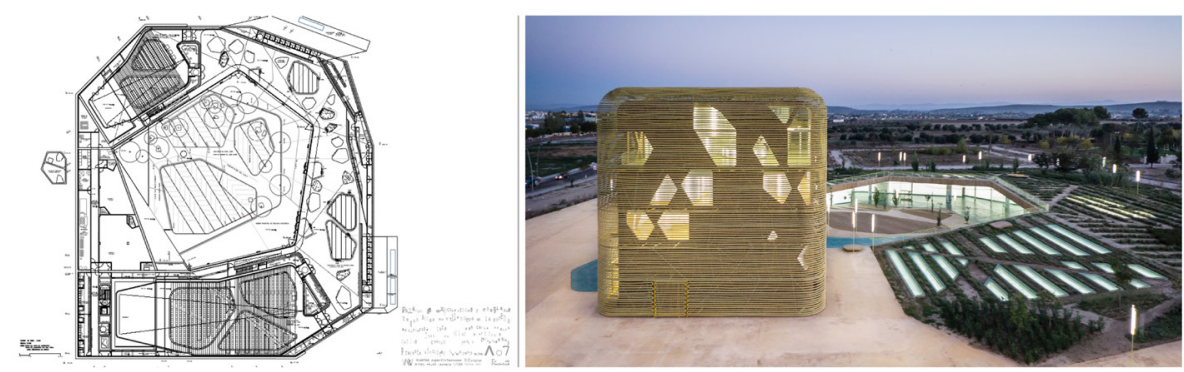

Figura 7. Luis Pancorbo, José de Villar, Carlos Chacón e Inés Martín Robles, Palacio de Congresos Vegas Altas, Villanueva de la Serena. Planta: cortesía de Pancorbo Arquitectos. Foto: CJesús Granada, cortesía de Pancorbo Arquitectos. 

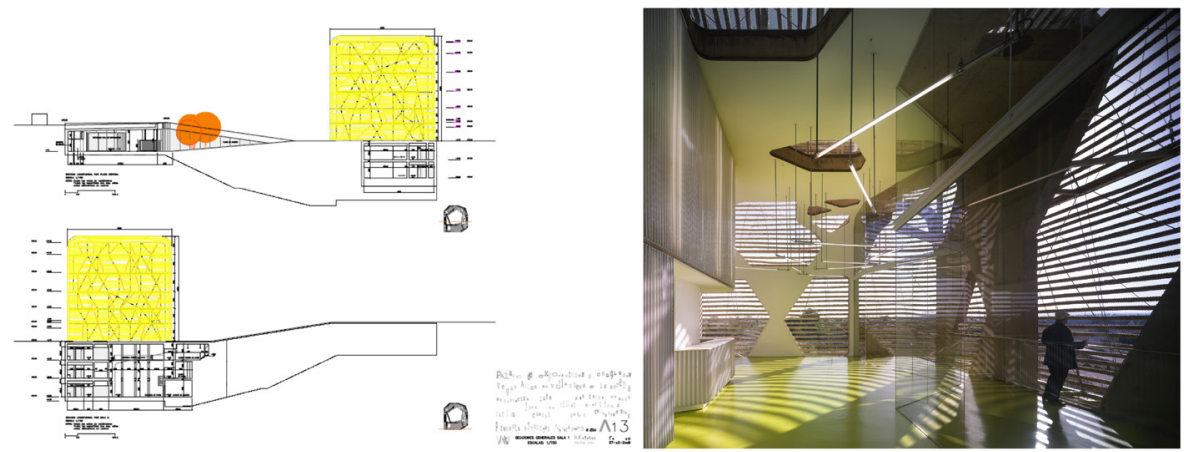

Figura 8. Luis Pancorbo, José de Villar, Carlos Chacón e Inés Martín Robles, Palacio de Congresos Vegas Altas, Villanueva de la Serena. Secciones: cortesía de Pancorbo Arquitectos. Foto: @Jesús Granada, cortesía de Pancorbo Arquitectos. 